\title{
Expression of K-Ras, p53 and Ki-67 in Precancerous and Cancerous Lesions of Colorectum
}

\author{
Sophia Thomas ${ }^{1}$, Arvind Bhake ${ }^{2}$ \\ ${ }^{1}$ Department of Pathology, Jawaharlal Nehru Medical College, Datta Meghe Institute of Medical \\ Sciences (Deemed to Be University). Sawangi (Meghe), Wardha, Maharashtra, India. \\ ${ }^{2}$ Department of Pathology, Jawaharlal Nehru Medical College, Datta Meghe Institute of Medical \\ Sciences (Deemed to Be University). Sawangi (Meghe), Wardha, Maharashtra, India.
}

\section{ABSTRACT}

\section{BACKGROUND}

The immunological assessment at molecular profiling of K-Ras, p53 and Ki-67 along with EGFR, BRAF and others like BCL-2 has been the focus of several workers. The incidence of colorectal adenomatous polyp with dysplastic changes and colorectal carcinoma per se in the context of Indian population as per the ICMR registry, New Delhi, India, is significant. We wanted to study precancerous lesions (adenoma, polyp) and adenocarcinoma of colorectum for the expression of K-Ras, p53 and Ki-67 in biopsy or surgical specimen of colon by immunohistochemistry for its occurrence. We also wanted to study the intratumor heterogeneity of aforesaid expression for the purposes of prognostication and correlation with other clinicopathological parameters.

\section{METHODS}

This is a cross sectional study conducted in the Surgical Pathology and Division of Immunohistochemistry, Department of Pathology, Jawaharlal Nehru Medical College, Sawangi (Meghe), Wardha, Maharashtra, India, for a duration of 2 years. Total seventy patients of all ages and genders suspected of 1) precancerous lesions of adenomas and polyps (35 cases) and 2) adenocarcinoma on colonoscopic biopsy or surgical specimens (35 cases) were included in the study. The expression of K-Ras, p53 and Ki-67 as primary objectives along with EGFR, BRAF and others like BCL-2 as secondary objectives were recorded, tabulated and compared for the purposes of predictive parameters in colorectal neoplastic lesions.

\section{RESULTS}

Most of the cases of CRC were over the age of 50 years and Male:Female ratio was 5:3. Twenty-five cases of group 2 (CRC) showed positive IHC for K-Ras. p53 was detected in 28 cases and high Ki-67 index was observed in all 32 cases of CRC. The comparative statistics in the pilot study showed that the immunoexpression of K-Ras, p53 and Ki67 is significantly higher in group 2 (CRC) compared to cases in group 1 (polyps) and group 3 (normal).

\section{CONCLUSIONS}

Expression of K-Ras, p53 and Ki-67 is likely to be the distinguishing tissue biomarkers between benign and malignant colorectal disease process as well as have prognostic and predictive value in colorectal cancer. The addition of EGFR, BRAF and BCL-2 would help in determining the pathogenesis and offer targeted therapeutic intervention.

\section{KEY WORDS}

K-Ras, p53, Ki-67, Adenocarcinoma, Adenomas, Polyps, Precancerous Lesions, Colorectum
Corresponding Author:

Sophia Thomas,

Junior Resident,

Department of Pathology,

Jawarharlal Nehru Medical College, Datta Meghe Institute of Medical Sciences, Deemed to be University, Sawangi, Meghe, Wardha, Maharashtra, India.

E-mail:drsophiathomas93@gmail.com

DOI: $10.14260 /$ jemds/2020/490

How to Cite This Article:

Thomas $S$, Bhake A. Expression of K-RAS, p53 and Ki-67 in precancerous and cancerous lesions of colorectum. J. Evolution Med. Dent. Sci. 2020;9(32):22612265, DOI: $10.14260 /$ jemds/2020/490

Submission 04-02-2020,

Peer Review 04-07-2020,

Acceptance 10-07-2020,

Published 10-08-2020.

Copyright (C) 2020 JEMDS. This is an open access article distributed under Creative Commons Attribution License [Attribution 4.0 International (CC BY 4.0)] 


\section{BACKGROUND}

Despite immense advancements in cancer research, colorectal cancer still remains a dreaded disease. The therapeutics has certainly lowered cancer related morbidity and mortality. Still, the rate of survival is low and mortality remains high even after the target specific treatments. ${ }^{(1)}$

Conceptual change has been felt over a past decade by the workers engaged in the studies of colorectal lesions of colorectal carcinoma (CRC) and colorectal adenomas with glandular dysplastic changes. CRC in past few decades have been the objective of research in medical sciences all over the world. Its aetiology and risk factors have widely been studied which includes the dietary habits, molecular abnormalities, cell signalling pathways and others.

Evidence generated through the multiple molecular studies have implicated that the lesion of colorectal adenomas with dysplasia over a period of time changes to histological lesions of colorectal carcinoma. The molecular basis of it has been explained in a few studies. The biomarkers in evaluation of colorectal carcinoma has been published by American Society of Clinical Pathology, College of American Pathologists, Association of Molecular Pathologists and American Society of Clinical Oncology. The summary of guidelines speaks volumes about molecular assessment of CRC not only for its diagnosis but also for its prognosis and therapy outcomes. (1-10)

The deliberations have come to the conclusion that the molecular studies for certain mutated genes and products is essential as it enables targets for the treatment, distinction between benign, dysplastic, and atypical colorectal lesions from colorectal adenocarcinoma. It also predicts tumour behaviour and the prognosis and therapeutic outcomes. (7)

The immuno-molecular assessment of biopsies at molecular profiling of K-Ras, p53, Ki-67 along with EGFR, BRAF and other like BCL-2 is the focus of investigation by several research workers. The incidence of colorectal adenomatous polyp with dysplastic changes and colorectal carcinoma per se in context to Indian population as per the ICMR registry, New Delhi, India is significant. According to the Global Burden of Disease Study 1990-2016, India State-Level Disease Burden Initiative Cancer Collaborators, Lancet Oncol 2018; 19: 1289-306, the incidence of colorectal carcinoma is $5.8 \%$. Colorectal cancer is the $3^{\text {rd }}$ to $5^{\text {th }}$ leading cause of cancer deaths globally.

The molecular profiling of CRC by now is practised in specialised laboratories only who have the facilities of immunohistochemistry (IHC) and molecular biology. The data world over in context to the molecular profiling of CRC shows that such profiling is a much needed exercise to treat CRC. Not only therapeutics but also the predictive analysis at the outcome of the treatment is also based on molecular profiling of tumour cells of CRC.

There are few similarities and dissimilarities of molecular expression between atypical adenomatous lesions of colorectum when compared to colorectal cancer cells. But adenocarcinoma and atypical adenomatous lesions of colorectum has been inadequately dealt for the molecular expressions in published Indian medical literature.

The conventional prognostic and predictive parameters for CRC are the gross types, locations, age, histological types, histological grade, level of invasions, nodal metastases and distant metastases. These clinicopathological parameters however is of little use for therapeutic interventions. The subcellular signalling pathway molecules are of immense help not only for distinguishing benign versus malignant but also targeted therapeutic interventions. There are studies which analysed the expression of K-Ras, p53 and Ki-67 along with EGFR, BRAF and others like BCl-2. The IHC for these molecules have been found useful in distinguishing atypical benign versus malignant colorectal lesions. However, the correlation of these molecular expressions with foresaid clinicpathological parameters have been performed rarely. The Indian Medical literature is scantily referring to this aspect of the research in colorectal pathology. $(1,2,5,6,8,9,10,11,12,13,14,15)$

The research workers across the globe are actively engaged in exploring cell signalling pathways of CRC cancer cells to bring out ideal therapeutic options.

The IHC is a superior and yet simple technique that demonstrates the presence versus absence and locations of a particular type of wild versus mutated molecule of cell signalling pathway with high efficiency especially in neoplastic colorectal lesions.

Therefore, the research question set in for the present study is as follows- "Do the expression of K-Ras, p53 and Ki-67 distinguish between an epithelial precancerous and cancerous lesion and is their expression correlates with conventional diagnostic and prognostic clinicopathological parameters?"

The study hypothesis states expression of K-Ras, p53 and Ki-67 are associated with clinicopathological features that determine the biological behaviour, prognosis and treatment outcome of epithelial precancerous and cancerous lesion of colorectum.

\section{Objectives \\ The present study titled "Expression of K-Ras, p53 and Ki-67 in precancerous and cancerous lesions of colorectum" is being carried out with the objective to study precancerous lesions and the adenocarcinoma of colorectum for the expression of $\mathrm{K}$ - Ras, p53 and Ki-67 in biopsy or surgical specimen of colon by immunohistochemistry (IHC) methods and to know the intratumor heterogeneity of a foresaid expression.}

\section{METHODS}

This is a cross-sectional study conducted in the Surgical Pathology and Immunohistochemistry division of Department of Pathology, Jawaharlal Nehru Medical College, Sawangi (Meghe), Wardha, Maharashtra, India for a duration of 2 years. A total of 70 patients' biopsies or surgical specimens irrespective of age and gender were included in the study. Sample size was taken for the convenience.

of 70 cases, thirty-five cases diagnosed as precancerous villous adenoma, tubulo-villous adenoma, tubular adenoma, serrated adenoma and polyps were included in Group 1. Group 2 included thirty-five cases of adenocarcinoma diagnosed on colonoscopic biopsy or surgical specimens of colon and Group 3 - Control group consisted of 35 cases of colorectal biopsies which were reported as normal histomorphology. The study included prospective and retrospective retrieval of the cases 
of past 2 years diagnosed on biopsy and surgically resected specimens.

\section{Inclusion Criteria}

The cases diagnosed as Adenocarcinoma on colonoscopic biopsy and surgical specimens. ii) The cases diagnosed as precancerous lesions of serrated adenoma, tubular adenoma, tubule-villous adenoma, villous adenoma and adenoma with dysplastic changes on colonoscopic biopsy and surgical specimen.

\section{Exclusion Criteria}

1. The cases with non-availability of clinicopathological data

2. The cases which are reported as suspicious for colorectal malignancy on inadequate biopsies.

The comparison of expression of K-Ras, p53, Ki-67 is being carried out as follows: Precancerous vs cancerous lesions of colorectum, intratumoral heterogeneity with the scores and objective based clinicopathological features.

\section{Technical Considerations for Surgical Specimen}

- Gross examination and tissue sections of surgical specimens is being carried out by standard methodologist as described in standard text.(16,17)

- The tissue section processing is to be carried out by standard protocols of tissue processing. $(16,17)$

- Assessment of biopsy for histomorphological features is being performed by utilizing standard textbooks and reference books over the diagnosis of lesions.(16)

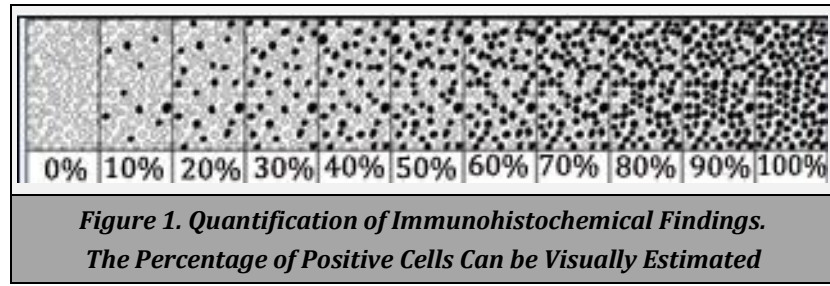

Immunohistochemistry Analysis for K-Ras, p53, Ki-67: $(1,2,5,6,8,9,10,11,12,13,14,15,16,19)$

- This study is being performed in 3 step method by available commercial antibodies based on Immunoperoxidase method.

- Monoclonal antibodies (are used to detect expression of K-Ras, p53, Ki-67) on 4 mm thick tissue section. (K-Ras: ab180772, abcam, p53: cloneD0-7 DAKO, Ki-67: DAKO)

- The results are expressed for negative and positive interpretation for expressed K-Ras, p53, Ki-67 on IHC with determined percentile of cells, intensity of staining, location and score as put forth by Fitzgibbons PL et al.(19)

- The positivity and scores are expressed for K-Ras, p53, Ki67 on IHC with determined percentile of cells and intensity of discolouration and location as put forth by references.

- $\quad$ Scale used for quantification of IHC (Figure 1).

\section{Ethical Issues}

No ethical issues are involved with the present study as the study is carried out on biopsy and surgically resected specimen, therefore do not risk or harm the human subjects. The confidentiality of the patient is maintained as per the hospital policy and ethics. As per the hospital policy, the consents are obtained before minor and major surgery as a protocol by surgical department.

IEC number of the present study is IEC /18-19/7459.

\section{Short Review of Literature}

Lu et al (7) studied the role of p53 and K-Ras in the diagnosis of early CRC. The study was carried out as a single centre analysis. The study comprised of 801 colorectal polyps resected on endoscopically. The expression of K-Ras, Ki- 67 and p53 were detected by IHC. The histological analysis was done on sections stained by $\mathrm{H}$ and $\mathrm{E}$. The variables in the study were compared by Pearson's test, Spearman test and One Way Anova. The study observed that, the condition of polyp was observed in the patients more than the age of 50 in $90.76 \%$ of patients. The IHC for K-Ras showed the involvement to be significant. ( $\mathrm{p}$ value less than 0.01 ). The similar observations were made for p53. Ki-67 expression with high average score was observed in $54.3 \%$ of the cells. The study concluded that screening for the expression of p53 and K-Ras can guarantee the diagnosis of CRC in early stage.

Zlatian et al(2) did a study for immunohistochemical evidence for tumour heterogeneity in 50 patients of CRC. The objective of the study was to assess the expression profile of the proliferation feature of Ki-67, K-Ras and p53 along with BCL2 expression. The mean age in this study was 59.7 years and there were 27 males and 23 females as the study subjects. The clinical pathological data of age, sex, location, histological type, tumour grade and others were recorded. The IHC over the tissue sections was performed by standard methods. Statistical univariate Anova analysis and Student's T test was performed along with Pearson's coefficient. The study observed that $52 \%$ (26 cases) express K-Ras and was expressed more with poorly differentiated adenocarcinoma. The p53 expression was observed in 43 (86\%) cases. Ki-67 was found to have a variable expression depending upon grade of adenocarcinoma. The Ki-67 index of $15-30 \%$ was observed with grade 1 , grade $225-60 \%$ and $50-90 \%$ in grade 3 . The average Ki-67 expression was in the range of $32.8 \%$ to $59.4 \%$. The study concluded that the expression profiles of Ki- $67, \mathrm{~K}-$ Ras and p53 had a significant positive correlation amongst them as well as CRC. The study further concluded that there exists intra-tumoral heterogeneity and existence of relationship between expression of foresaid molecules and other clinicopathological parameters.

Piton et al (20) studied K-Ras expression in CRC along with other mutations of BRAF. The study was carried out in 63 samples of formalin fixed paraffin embedded tumour specimens of CRC. The IHC was performed by standard methods using DAKO Envision system. The mean age of the patients was 63 years. The study concluded that the K-Ras and BRAF both can be detected on IHC with high specificity in CRC. The IHC especially for BRAF can be an alternate method for its detection in CRC instead of performing molecular biology techniques on it for its detection. 


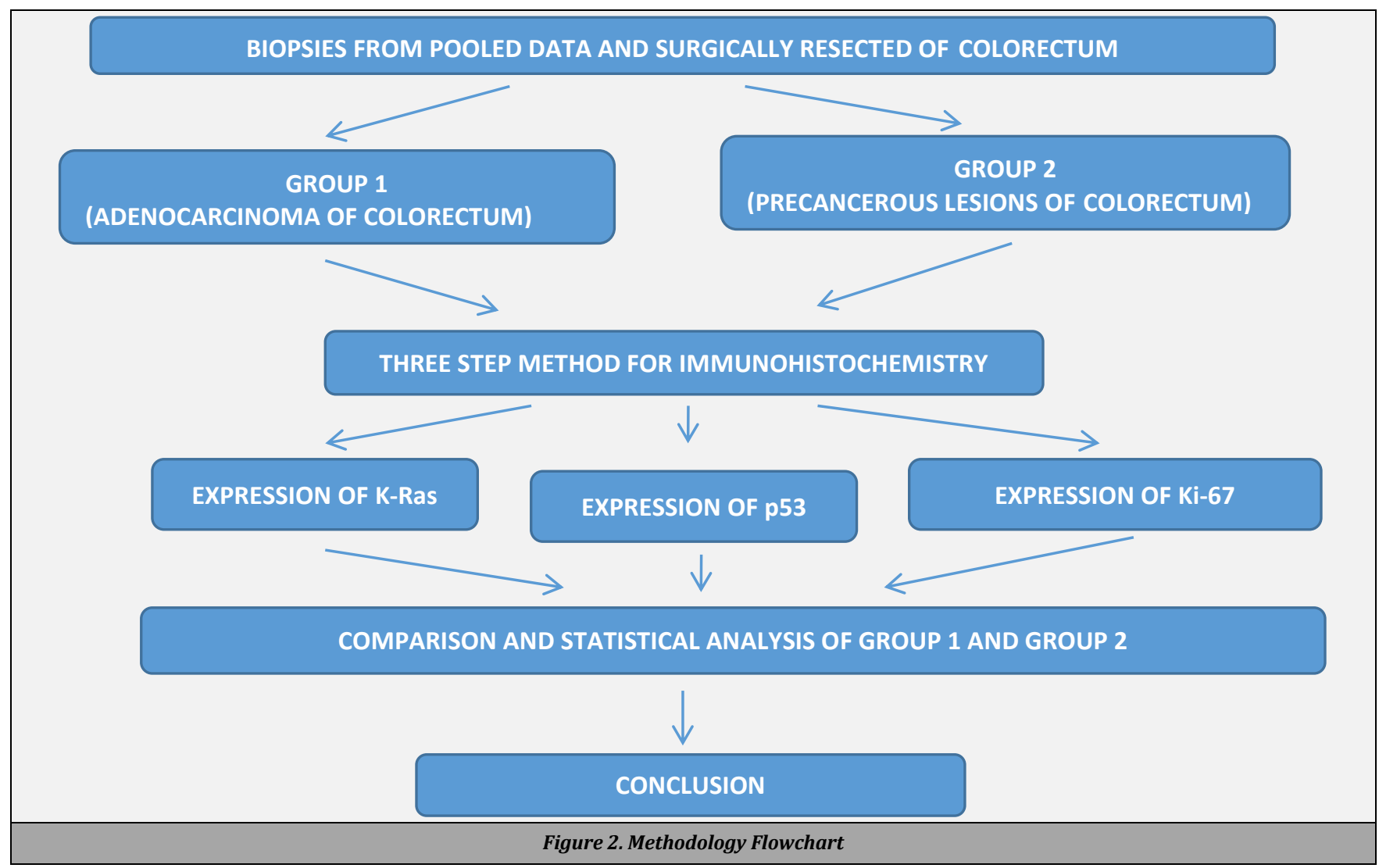

\section{Statistical Analysis}

Data was analysed using Student's T test and Pearson's coefficient.

\section{RESULTS}

Thirty-two specimens of CRC (group 2), 20 specimens of colorectal polyps (group 1) and 20 cases of normal colorectal mucosal biopsy (group 3).

- Most of the cases of CRC were over the age of 50 years and Male:Female ratio was 5:3.

- Twenty-five cases of group 2 (CRC) showed positive IHC for K-Ras. p53 was detected in 28 cases and high Ki-67 index was observed in all 32 cases of CRC.

- The comparative statistics in the pilot study showed that immunoexpression of K-Ras, p53 and Ki-67 is significantly higher group 2 (CRC) than cases in group 1 (polyps) and group 3 (normal).

- The study is in progress to correlate the finding of IHC of K-Ras, p53 and Ki-67 with clinicopathological parameters of tumour grade, histological type and Duke's and TNM stage of CRC.

- The intergroup and inter variable differences for expression of K-Ras, p53 and Ki-67 is being carried out.

\section{DISCUSSION}

It has been quoted in literature that K-Ras, p53, Ki-67 along with EGFR, $\mathrm{BCl} 2$ and BRAF V600E is expressed and overexpressed with CRC of poorly differentiated type.[3,6,8,12] Many studies suggested the intense expression of K-Ras to be considered as an independent prognostic feature. ${ }^{[3,10,12]}$ As KRas links with other clinicopathological features such as grade, stage and metastatic disease so it can be quoted as "More the K-Ras, poorer the prognosis" by literature. The discussion will be designed to compare the findings of the present study with the observations available in the different studies. $(3,6,8,10,12)$ The auxiliary observations performed over these objectives of EGFR expression will be compared with available observations in the literature for the conclusion of correlation of EGFR expression with grade and stage of CRC. The controversial observation do exists in the literature for EGFR expression by tumour cells of CRC.[2,9]A few studies concludes that EGFR expression is more with the poor grade of CRC.[8,9] EGFR expression has also been contradicted in a few other studies that it is independent of grade and stage of CRC. [5]

An adjunct discussion will be constructed over the topic of Anti-EGFR antibodies treatment which is a new find for treatment of CRC since a decade. ${ }^{[2,4,9]}$ It has been debated a lot in the medical literature for the outcome of Anti-EGFR treatment; tilting the balance in favour of this option because of encouraging results. ${ }^{\left[{ }^{[0]}\right.}$ Anti-EGFR treatment can be effective only if there exists a co-expression of relay protein of $\mathrm{K}$-Ras for execution of cell signalling pathway.[2] Mere EGFR detection on IHC could not pave way for the treatment until K-Ras too has been detected, is the proposed and actualized hypothesis in published literature. There are reports in literature over a sizeable number of CRC highlighting EGFR \& K-Ras detection and its importance for successful treatment outcome by AntiEGFR treatment specially in metastatic CRC.[7,8,20] The discussion will also be done for relationship of EGFR and K-Ras expression for various clinicopathological parameters. 


\section{CONCLUSIONS}

Biomarkers K-Ras, p53 and Ki-67 help in distinguishing benign from malignant colorectal disease process and also work well as prognostic and predictive parameters for colorectal cancer. The IHC of these biomarkers would help in targeted therapeutic interventions.

Small sample size was a limitation of the study.

Financial or Other Competing Interests: None.

\section{REFERENCES}

[1] ICMR. Registry of colorectal cancer.

[2] Zlatian MO, Comanescu VM, Rosu FA, et al. Histochemical and immunohistochemical evidence of tumor heterogeneity in colorectal cancer. Rom J Morphol Embryol 2015;56(1):175-81.

[3] De Hertogh G, Gerboes PK. Practical and molecular evaluation of colorectal cancer: new roles for the pathologist in the era of targeted therapy. Arch Pathol Lab Med 2010;134(6):853-63.

[4] Johnston PG. Identification of clinically relevant molecular subtypes in colorectal cancer: the dawning of a new era. The Oncol 2014;19(5):568-73.

[5] Azarhoush R, Heidari K, Samadzadeh S, et al. Expression of p53 protein in colorectal cancer and association with prognostic factors in Northeast Iran. Acta Medica Iranica 2018;56(3):161-5.

[6] Lumachi F, Orlando R, Marino F, et al. Expression of p53 and Ki67 as prognostic factors for survival of men with colorectal cancer. Anticancer Research 2012;32(9):39657.

[7] Lu YH, Lin RT, Zhou GX, et al. Critical role of p53 and KRAS in the diagnosis of early colorectal cancer: a oneyear, single- centre analysis. Int J Med Sci 2017;14(11):1154-62.

[8] Sepulveda RA, Hamilton RS, Allergra JC, et al. Molecular biomarkers for the evaluation of colorectal cancer: Guideline from The American Society for Clinical Pathology, College of American Pathologists, Association for Molecular Pathology and American Society of Clinical Oncology. Arch Patho Lab Med 2017;141(5):625-57.
[9] Ahmed YN, Ismail TA, Kareem ST. A clinicopathologic study of Ki-67 proliferation index in colorectal carcinoma. Saudi Med J 2012;33(8):841-5.

[10] De Sousa WAT, Rodrigues LV, Da Silva RG Jr, et al. Immunohistochemical evaluation of p53 and Ki-67 proteins in colorectal adenomas. Arq Gasteroenterol 2012;49(1):35-40.

[11] Samowitz SW, Curtin K, Khe-ni Ma, et al. Prognostic significance of p53 mutataions in colon cancer at population level. Int J Cancer 2002;99(4):597-602.

[12] Petrisor 0, Guisa S, Sajin M, et al. Ki-67, p53 and bcl-2 analysis in colonic versus rectal adenocarcinoma. Rom J Morphol and Embryol 2008;49(2):163-71.

[13] Rambhau P, Odida M, Wabinga H. p53 expression in colorectal carcinoma in relation to histopathological features in Ugandan patients. African Health Sci 2008;8(4):234-8.

[14] Liljegren A, Lindblom A, Rotstein S, et al. Prevelance and incidence of hyperplastic polyps and adenomas in familial colorectal cancer: correlation between two types of colon polyps. Gut 2003;52(8):1140-7.

[15] Sepulveda RA, Hamilton RS, Allergra JC, et al. Molecular biomarkers for the evaluation of colorectal cancer: Guideline Summary from the American Society for Clinical Pathology, College of American Pathologists, Association for Molecular Pathology and American Society of Clinical Oncology. J Oncol Pract 2017;13(5):333-7.

[16] Rosai J. Rosai and Ackerman's Surgical Pathology. Vol. 1. $10^{\text {th }}$ edn. Mosby 2011.

[17] Suvarna K. Bancroft's Theory and practice of histological techniques E - book. 7th edn. Churchill Livingstone 2012.

[18] Mohandas KM. Colorectal cancer in India: controversies, enigmas and primary prevention. Indian J Gastroenterol 2011;30(1):3-6.

[19] Fitzgibbons PL, Bartley AN, Connolly JL. Template for reporting results of biomarker testing of specimens from patients with carcinoma of the breast. Breast 2018;1:1.

[20] Piton N, Borrini F, Bolognese A, et al. KRAS and BRAF mutation detection: Is immunohistochemistry a possible alteranative to molecular biology in colorectal cancer? Article ID 753903, Gastro Res and Pract 2015;2015:8. 